\title{
Internalization, Qualitative Methods, and Evaluation
}

\author{
Sarah Faisal ${ }^{1}$, Brock Craft ${ }^{2}$, Paul Cairns ${ }^{3}$, Ann Blandford ${ }^{1}$ \\ ${ }^{1}$ University College London \\ Remax House, 31/32 Alfred Place \\ ${ }^{2}$ The London Knowledge Lab \\ ${ }^{3}$ University of York \\ 23-29 Emerald Street \\ London WC1E 7DP, UK \\ +44 (0) 2072076795225 \\ London WC1N 3QS \\ +44 (0) 2077632137 \\ \{s.faisal, a.blandford\} \\ b.craft@ioe.ac.uk
Dept of Computer Science
York YO10 5DD, UK
+44 (0)1904 434751 \\ pcairns@cs.york.ac.uk
}

@cs.ucl.ac.uk

\begin{abstract}
Information Visualization (InfoVis) is at least in part defined by a process that occurs within the subjective internal experience of the users of visualization tools. Hence, users' interaction with these tools is seen as an 'experience'. Relying on standard quantitative usability measures evaluates the interface. Yet, there is more to users' interaction with InfoVis tools than merely the interface. Qualitative methods targets users' subjective experiences. In this paper we demonstrate the potential benefits of qualitative methods, more specifically Grounded Theory, for generating a theoretical understanding of users' InfoVis experiences through discussing the results of a qualitative study we conducted. The study was conducted in order to evaluate a visualization of the academic literature domain, which we have designed and built using a user-centered design approach. The study resulted in us identifying categories that are essential to the InfoVis experience. This paper argues that these categories can be used as a foundation for building an InfoVis theory of interaction.
\end{abstract}

\section{Categories and Subject Descriptors}

H5.m. Information interfaces and presentation (e.g., HCI): Miscellaneous.

\section{Keywords}

Qualitative Methods, Information Visualization, Usability, Evaluation

\section{INTRODUCTION}

From the user's perspective Information Visualization (InfoVis) is not just the externalization of data. In fact it is more of an internalization process that users engage with in order to make sense of the externalized visual representation of a domain. A user's interaction with Information Visualization (InfoVis) tools is an experience that goes beyond the interface. Standard quantitative usability measures merely address the usability of the interface without tapping into the subjectivity of the experience. Since users' interaction with InfoVis tools is a subjective experience which is reflected through the internalization of the data, we are going to need more than usability. In fact, we need to rely on methods that tap into the subjectivity of such an

Permission to make digital or hard copies of all or part of this work for personal or classroom use is granted without fee provided that copies are not made or distributed for profit or commercial advantage and that copies bear this notice and the full citation on the first page. To copy otherwise, or republish, to post on servers or to redistribute to lists, requires prior specific permission and/or a fee.

$B E L I V$ '08, April 5, 2008, Florence, Italy.

Copyright 2008 ACM 978-1-60558-016-6/08/0004 ..\$5.00. experience.

Qualitative research methods aim at understanding the user, not through preset questionnaires or tasks, as do the quantitative usability measures. However, they aim at generating theories that are based on current situations. In this paper we will show that by applying a qualitative research method, Grounded Theory (GT), that we were able to take a step further into understanding users' InfoVis experiences, which has not previously been attempted. Through the GT analysis we were able to identify categories that have a direct effect on the InfoVis experience. These categories can be seen as a starting point to forming the foundation of an InfoVis theory of interaction. We strongly believe that such a theoretical grounding is essential for devising evaluation methodologies that are tailored to InfoVis tools. The data that we analyzed was based on semi-structured interviews which we conducted with the users after their interaction with a visualization of the Academic Literature Domain (ALD). This tool was developed using a User-Centered Design (UCD) approach. We conducted the study with a total of 12 users. However, as we are using a qualitative research methodology, GT, this study is still ongoing with analysis interspersing interviewing. The results of the analysis affect subsequent interviews. This will continue until a saturation point is reached. Hence, we will present the results of analyzing the data of 6 users, we are currently still in the process of analyzing the rest of the interviews. The results of the latter will determine whether or not a saturation point has been reached.

The aim on this paper is to demonstrate the benefits that GT offers to the evaluation of InfoVis tools. The power of this method lies in its ability in devising theory. This paper starts by revealing the reasons why InfoVis tools are to be considered as experiences and not merely interfaces. Following that an overview of the Academic Literature Domain (ALD) InfoVis will be given, which is followed by an overview of the usability study conduced. This study was conducted in order to evaluate the usability of the interface. An overview of qualitative methods and more specifically GT will be given will follow, followed by the experiential evaluation study conducted. The results, although still preliminary, we believe are ground breaking since they point to some aspects of the InfoVis experience that are crucial and yet have not been previously addressed.

\section{The InfoVis Experience: Internalization vs Externalization}

Visualization has become a technology-oriented field, where images are represented out of the mind and upon computer screens. InfoVis tools visually represent abstract data of a particular domain in order to assist users to gain knowledge and insight of the represented domain. Spence [21] defines this as the 


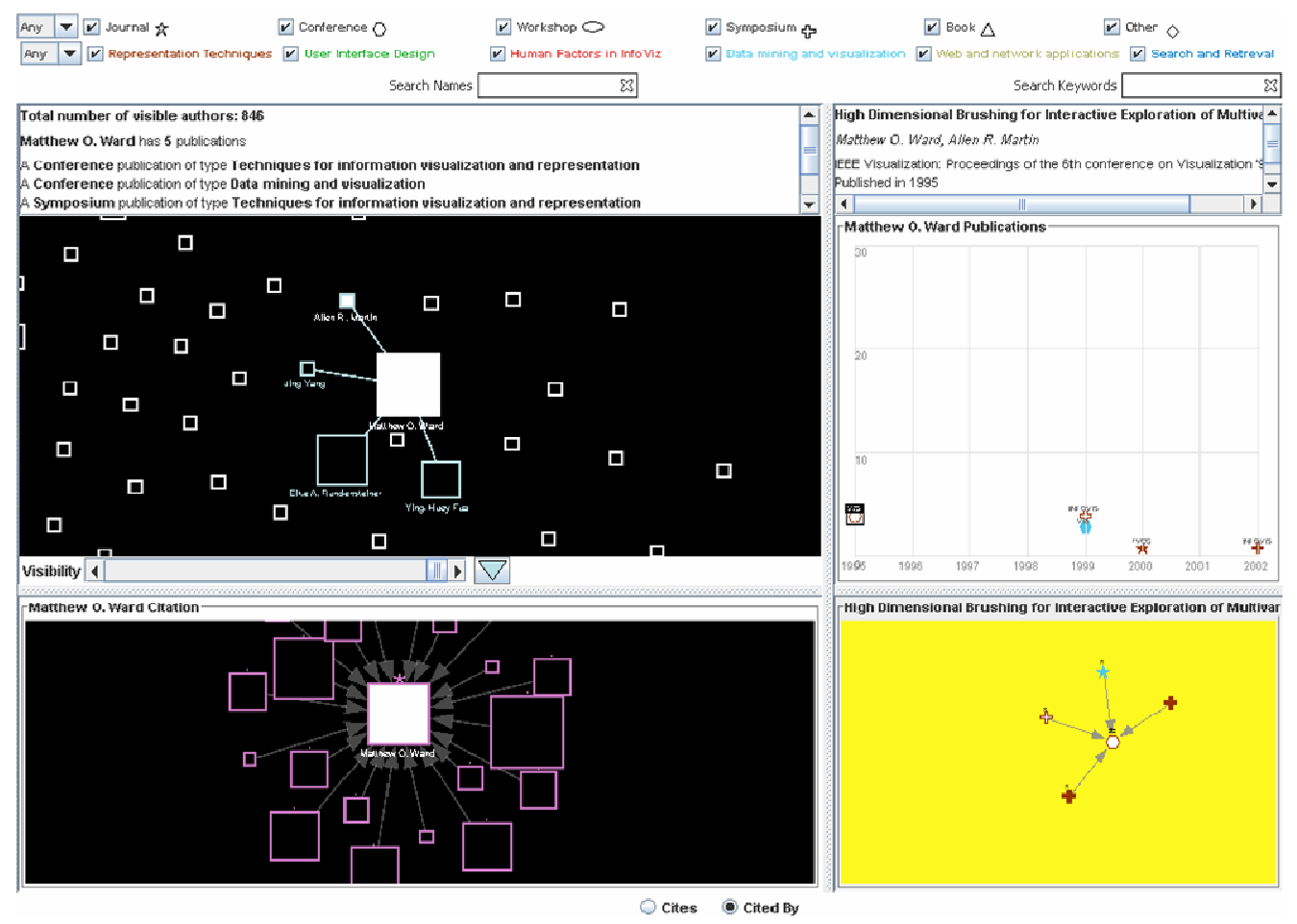

Figure 1. Academic Literature Visualization Tool

externalization of the data. Determining externalization is the main role of the designer. The opposite of externalization is internalization. The internalization process is the main role of the InfoVis user. It results from users' interaction with the external representations. Consequently, users start to make sense of the represented concepts and hence build internal models of the domain [23]. Hence, from the users' perspective, visualization is more than technology: it is in itself an activity, more precisely a cognitive activity with which users engage [13]. Recognising the cognitive dimension, Spence [21] defines visualization as a cognitive activity which involves the formation of mental images, and Card, et al. [3] refer to visualization as "amplifying cognition".

Users employ these tools to satisfy their differing needs and goals, which might be to gain insight and knowledge or to test specific hypotheses. It is through users' interaction with the tool that they are able to satisfy these goals. In other words, it is this interactivity that allows for a subjective user experience [9]. As the users interact with the tool, "ah HA!" moments arise [3][21]. How and when these moments are reached differs from one user to another. It is these "ah HA!" moments that we believe makes them a subjective experience since it is something that is unpredictable and hence particular to each person.

Both InfoVis literature and HCI literature refer to cognitive activities that users engage with in order to gain knowledge and interact with a system. However, there are key differences. In the InfoVis literature, internal models represent the models that people construct of a particular domain, whereas in HCI, it represents the models that people develop of the interface [17]. When it comes to internal models, in the context of InfoVis, there is no right or wrong: the question is how people make sense of something they interact with. Since such images are personal and internal, they are not open to rigorous testing. Capturing and validating mental models is a difficult task due to their subjectivity. We believe that the challenges of evaluating InfoVis tools are based upon the fact that standard HCI usability measures are designed to capture the usability of the interface and not move beyond that, whereas the focus when evaluating InfoVis tools should somehow tap into users' internalization process. In other words, we need to be able to determine whether or not the visualization is able to assist users in building domain-related mental models. Hence, what we really need is a theory of InfoVis interaction.

During the course of our research we developed and evaluated the interface of the ALD InfoVis tool, as usability is an essential part of the experiences. The evaluation took the form of a standard usability study.

\section{The ALD InfoVis Tool: Design and Evaluation}

The main goal of our research is focused towards understanding and capturing user's InfoVis experience. In order to do so, we developed an information visualization of the ALD. The development process was a User-Cantered Design (UCD) one. Requirements were developed through qualitative interviewing [8] and these led to a design based explicitly on these requirements [10] (Figure 1).

\subsection{Requirements and Design}

An academic literature domain comprises all the data on the literature covering a particular field of study, e.g.: authors, papers, citations and so on. The intended users are academics who are not 
only progressing in their own research but also developing and monitoring their understanding of other work being done in the field. Literature data is complex due to its size and the interrelations that exist between entities. From our requirements assessment [8], it became clear that users equate authors with their publications and position authors in terms of the work that they build on through citation. These became key design motivations. Moreover, users make sense of the literature through constantly revealing more details and seemed to spend more time interacting with the details of literature rather than forming "bigger pictures."

The interface was divided into 4 views as seen in Figure 1. The main view is the authors' view, which is the bigger view on the top left; this is due to the fact that the members of the community are the center of the sense making activity, as revealed by the results of the qualitative study. The publication view reflects the details, since publications are produced by the authors, seen on the top right. As the user selects an author, all the author's papers are displayed on the paper view. The qualitative study revealed that citation links that are of interest to the users are not just, as we might assume, between the publications, but also between authors. Hence, we included the authors' citation view which represents the citation information between authors located in the bottom left of the screen, and the publication citation view, which represents citation information between publication, located in the bottom right corner of the screen. Users are able to drag entities between the various views and view the relationship that the view represents. For example, the user can drag a publication into the publication citation view and see, as a result of that, all the publications that cite or are cited by that particular publication. Similarly, by dragging an author citation link into the publication citation view the user can see the details of that relationship. For example: if author1 cites author2 in the author citation view and the user drags the citation link into the publication view then the user will see all papers of author1 which cite papers of author2. The interface is described in more detail in Faisal et al [10]. The data being visualized was from the InfoVis'04 contest which includes the complete metadata of 8 years for all InfoVis conference papers and references from 1995 to 2002 [11]. This data included information such as: author names, papers titles, abstracts, keywords, and citation and collaboration information.

Before going on to explaining the usability study, we would like to briefly point to a tool that we used in our prototype, which we call the "marking tool". This tool simply allowed users to highlight entities such as authors and publications. This functionality, although quite simple, resulted in an unanticipated reaction from the users which we will be discussing later on.

\subsection{Usability Study}

The aim of this study was to evaluate the usability of the interface. A set of low-level tasks were devised that targeted the visualization's visual cues. This idea was inspired from a prominent evaluation study in the field by Morse et al [15] where they devised tasks based on visual taxonomies. Tasks were ranked in three levels of difficulty as detailed below. The difficulty levels were based on the primitiveness of the actions that the users had to engage with in order to accomplish the task.

Primitive tasks represent the basic tasks needed to interact with the interface such as locate and identify. Intermediate tasks represent tasks that are executed through a combination of primitive tasks. And last but not least, complex task, are highlevel tasks since they are based on users' exploration of the visualization and not on specifics. They are less controlling compared to the primitive or the intermediate tasks in terms of the actions that the user must execute, and the answers they provide. For example: What is the relationship between the following authors [author name] and [author name]? Since the grounds of the relationship are unspecified, different users can reach different results in various ways.

\subsubsection{The Study}

The evaluation was a lab-based evaluation centered on 22 tasks covering the range of complexity described. The tasks were presented in order of difficulty reflecting cumulative familiarity and knowledge of how the interface worked. Users were timed and their answers were collected. In addition, researcher's observations were gathered. Seven participants were used, as we felt that this would begin to reveal major usability problems [16] and also allow us to explore the mismatch with a "proper" InfoVis evaluation. All participants had at least two years of research experience. Prior to beginning the study users were given fifteen minutes training where the system was explained to them and participants could perform some training tasks akin to those used in the study. After conducting the study, participants were asked to answer a questionnaire which was based on the Questionnaire of User Interface Satisfaction (QUIS) [4], in addition to commenting on the tool.

\subsubsection{Results}

This study was successful in that it assisted us in identifying some usability problems. In addition, the study showed that usability measures: effectiveness, efficiency and user satisfaction stopped at the interface and hindered what was beyond, that is, the subjectivity of the experience.

Effectiveness- All users got most of the answers correct with the highest scoring $96 \%$ and the lowest scoring $87 \%$. This indicates that the visual cues used by the system were understandable by the users.

Efficiency- Time was not a suitable measure since users took varying paths whilst executing the tasks, especially when it came to the high-level tasks. For example, users were given a task to identify the relationship between two authors; one user accomplished the task in $16.3 \mathrm{sec}$ whereas another accomplished the task in $64.5 \mathrm{sec}$. Both users identified accurate relationships except that one took a longer path in examining the domain.

User Satisfaction- analysing the QUIS showed that the users had a neutral reaction towards the interface. Rating the system through previously designed questions does not reflect the feelings that the user has of the interactive experience.

We only got a glimpse of users' feelings through the comments that they gave, for example one of the users commented: "I think I would have a different appreciation of the system if it meant something and helped me", here the user indicates that his/her satisfaction was affected by their familiarity with the domain, which the QUIS failed to address.

This study was successful in identifying usability problems that were associated with the interface and the visual language used, in other words, the syntax. However, it said nothing about the knowledge gained. Rather, it seemed to hinder the experience. One user commented: "I liked the system but I was trying to complete tasks given - it might mean more if I used it for research and saw interesting relations in papers that was meaningful to 
$m e$ ”. Users were so focused on the tasks that "ah HA!" moments were never reached.

As a result, we needed to evaluate the tool through tapping into the internalization process, in other words understanding what is going on in users' heads and the experiences that they are generating. We are in the process of conducting a study where we give the users a non-restrictive high-level task. Following that, users are interviewed and the data is analyzed using the Grounded Theory methodology. However, before we go into detail in explaining the study, we will give a brief overview of qualitative research methods, more specifically Grounded Theory, as it is the method used in our study.

\section{Qualitative methods}

Cresswell [6] identifies five traditions of qualitative research design. These are: biographies, phenomenological studies, Grounded Theory (GT) studies, ethnographies, and case studies. From these traditions, many qualitative evaluation methods have been applied to usability assessment and the information systems design process. Ethnographic techniques and case studies have received particular attention. Many adaptations of these inquiry techniques have also been used, in particular, contextual interviews, observational studies, and contextual interviews, focus groups, and think-aloud approaches [2]. GT and Action Research [1] have also been applied, though they receive less attention, in general. We focus on GT as a method of evolving a rich, datagrounded account of the user-experience.

The grounded theoretical approach, or more often, modified techniques based on GT, have received attention in the realm of HCI. GT was developed in the realm of the social sciences as a means of theory development. This is in contrast to methods of theory testing, which have informed the design of traditional usability studies. It is used in situations wherein the researcher seeks to understand phenomena, with an aim to developing knowledge about them and formulating a theory. Instead of starting with a theory a priori, and testing it, the researcher develops a theory from evaluating the data. A thorough account of GT is provided in [22].

As applied to usability evaluation, the GT approach primarily involves the collection and analysis of interview or transcription data from users. This data is collected during requirements gathering, at design time, during usability testing, and with posttest interviews. Using this data, the researcher analyses the text, assigning meaningful open codes to related categories of data. Categories are a principal organizing method for theory development in GT. The categories are drawn from associations of information within the data itself in the form of recurrent themes. For example, during interviews, many participants may speak of their subjective experiences using the mouse for navigation in an information space. Similarities among their descriptions may point toward attributes of common experiences among the participants. Drawing from this data, the researcher can unite these common experiences to form a category from which theoretical propositions about the user experience can be made. Such propositions are conjectures about the user's subjective experience which are grounded in the data that was collected. The open codes are created, edited and refined until the researcher determines that further analysis will reveal little useful information, a point called saturation. The open codes can then be collected to form interrelated categories of information, in a process called axial coding. From these activities, the researcher develops an interpretation of the phenomena at work, from which theoretical propositions can be made. A rich set of categories can be used to identify overarching themes about the user experience.

Because the focus of GT is on developing theory about subjective experience, we argue that it also holds promise for understanding the subjective experience of using a visualization, primarily because this approach can illuminate the subjective, internal experience of the user and consequently can begin to reveal the factors surrounding "ah HA!" moments. It is a participatory method, which is aimed at eliciting internal dialogues and concerns. It allows for the development of emergent themes toward a theoretical proposition about subjective experiences, and this can be both quantified and grounded in data.

An example of using this approach would be to apply a GT approach to analyzing all phases of Visualization development including the Requirements Gathering Phase, the Design Activities Phase, and Usability assessment (i.e., analyzing think alouds). Although we know of no cases where GT has been applied to the analysis of visualization user experience, it has been applied to a case study of the design of visualization systems [5].

The visualization community has recognized the value of usability assessment in improving visualization systems, and there are an increasing number of reported usability tests. However, these reports are exclusively in the domain of the "traditional" methods of usability testing, and do not address the emerging emphasis that practitioners in the HCI community are placing on evaluating the qualitative and subjective. The authors are aware of a handful of studies reporting use of qualitative methods, for example, case studies [19], focus groups [14], and exploratory insight-based analysis [18] which explicitly evaluates visualization systems. In addition, Shneiderman and Plaisant [20] have advocated an ethnographically-based framework for evaluating the success of visualization systems.

A counter-argument to this approach is that the attributes of the internalization process may occur outside of the conscious awareness of users and thus, cannot be collected from user reports of their experiences. Accepting this problem, we believe that this approach is valuable because it will begin to describe the conscious accounts of user experiences (something which is currently lacking) and from these, common themes of discourse across users may yet point to unreported, but extant processes involved in internalization. Our approach may also provide a model for future research into the user experience of visualization systems.

More generally, one critique of qualitative methods is that they are subject to individual interpretation, researcher bias, and thus are not able to be easily generalized to a wide range of other cases and situations. This perspective contends that such studies lack validity. Acknowledging that there is room for debate about the merits of both traditions, it is not our purpose to engage in a debate about the nature of positivist versus post-positivist research paradigms. A thorough discussion of the issues is provided in [12]. Given the increasing number of qualitative studies in computer science and particularly in $\mathrm{HCI}$, we regard the qualitative and phenomenological research methods as having been accepted by the community at large.

\section{Capturing the Subjectivity of Experience}

Based upon this, we believe that qualitative research techniques can begin to illuminate the terrain of the subjective, internal 
experience of visualization users. In order to be able to tap into such subjectivity we conducted an experiential study in which we gave the users a high-level non-restrictive task. The reason for doing this was that we wanted the users to interact freely and naturally with the tool. After interacting with the tool, we asked users to talk about their experiences and feelings, and whether or not the tool was able to give them additional insight. This, in addition to users' observations will be the input of our qualitative analysis which we will do using GT. The results and the insight that we were able to gather from such a study were ground breaking in that they identified factors that are essential to the InfoVis experience which can be used as a foundation for a theory of users' InfoVis interaction. The study as presented here is still in progress so any results presented here are necessarily preliminary.

\subsection{The Study}

The study was setup in a lab-based environment. Six participants were given a high-level task which required them to explore the concept of Dynamic Queries and to try to identify the various research camps that target such a concept in addition to the key publications using the InfoVis tool described above. Participants were given a 1996 paper written by Shneiderman titled "Incremental Data Structures and Algorithms for Dynamic queries" as a starting point. However, they were told that they had the choice of whether or not to use this paper as their starting point. The users were told to interact freely with the tool and to stop whenever they felt that they had generated enough insight. Note that users had access to the titles, keywords, and the abstracts of the papers and not the actual paper. However, in certain cases some abstracts were missing from the dataset.

Prior to beginning the study, users were asked to fill in their demographic information in addition to rating their knowledge of InfoVis tools and the InfoVis'04 dataset. During the course of the study the researcher observed the participants, took notes of their interaction with the tool and assessed their experiences through their reactions and comments. User's comments during the interaction process were recorded in addition to screen interactions. Participants were also asked to rate their knowledge of the concept of Dynamic Queries, and if they were familiar they were asked to list the names of the researchers associated with that concept. This was intended to assess whether or not users were able to gain insight of the concept, in addition to whether or not their background knowledge affected the InfoVis experience.

After the study users were interviewed with a semi-structured interviewing process. We transcribed the interviews and analyzed them using the Ground Theory methodology. This resulted in the generation of categories through open and axial coding which started to hint towards a theory. However, we didn't feel that we had reached a complete saturation point as we were still refining the categories following the analysis of the interviews. As a result, we conducted the study with 6 more participants. The study took the same format as the first however; the interview questions were targeted towards validating our findings. This will continue until a saturation point is reached. In this section we will present the findings from analyzing the data of the first 6 participants. Even though a saturation point was not yet reached, our results nevertheless point to important information which will assist in the evaluation of future visualization tools. We will explain the categories which we identified that make up the user InfoVis experience. However, before explaining these it is essential to give an overview of the theory that is being generated.

\subsection{Overview of the Experience}

Users when engaging in an InfoVis experience tend to take on a systematic process to achieve an overall goal. This process is divided into tasks that are set by the users and are personal to their sensemaking needs. This reinforces the fact that preset-specific tasks are insufficient for evaluating InfoVis tools. Users who are unable to identify such a process tend to engage in a negative experience, as they become lost and hence are not working towards achieving a specific goal. While engaged in the sensemaking process users interact with the interface, which comprises on-screen widgets and physical input devices. In addition, users also interact with the data, which is reflected through the visual encodings and the insights that they generate. Any negative interference from any of these on the users' sensemaking activities will have a negative effect on the general experience. The ultimate goal of InfoVis is the transformation of the externalized visual representation into internalized mental models. This, as we have observed from our study, results in users' satisfaction. This satisfaction is affected by the flow of interaction and the functionality of the tool, in addition to users' backgrounds. Users' past experiences might have either a positive or a negative effect on their overall satisfaction.

\subsection{Categories and Concepts}

Five main categories were identified which are: flow of interaction, usability, insight, background, and satisfaction. It is the interconnection of these (Figure 2) that will take the user from externalization to internalization.

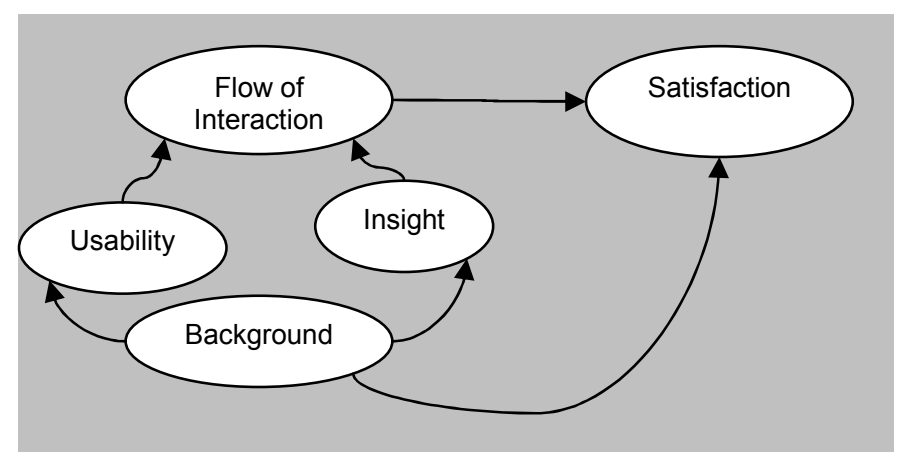

Figure 2. The InfoVis Experience

\subsubsection{Flow of Interaction}

The flow of interaction represents the main category that ties the InfoVis experience. Users interact with the tool to reach a specific goal, such as a hypothesis that needs to be validated, or specific insight that needs to be gained. In our particular case, the goal is to accomplish a high-level non-restrictive task in which users are required to learn about a specific concept: Dynamic Queries (DQ). As users interact with the visualization tool they engage in a systematic continuous process through which they work towards achieving the intended goal. For example, during the course of the study, one of the users who was asked about his experience said: "the general sense of geography...allowed me to be, what I thought to be relatively systematic".

Users accomplish these high level tasks through the execution of multiple low-level tasks that are personal to them and are related to their background and past experiences, "I tried to identify firstly key people and then maybe key concepts within the concept of DQ $I$ was trying to search for, and then tried to find papers 
afterwards". People are different, and so when it comes to designing for a subjective experience this subjectivity needs to be taken into account, as we did when gathering the requirements of the tool [8]. Hence, it is crucial to understand not only the domain, but also how people interact with such a domain, prior to designing the InfoVis tool. Each domain has its own identity and this needs to be reinforced as part of the design.

Two categories were identified during the course of analyzing our data that directly affects this flow of interaction, these are: usability and insight. Any negative influence from any of these categories will break the flow and hence will affect the experience negatively.

\subsubsection{Usability}

When users interact with the interface they engage with physical activities. These activities involve the use of physical input devices in order to interact with on screen widgets, such as clicking on a button or dragging a visual entity. In order for an interface to be usable, the system image must fit the user's model [17]. Usability is essential for achieving a positive user experience. One user, when asked to comment about the interaction said, "Most of the actions are similar to most of the other applications, ...even though there are lots of information given to me during the training I can just guess, like maybe right clicking [to get the popup menu]...because they are consistent with other applications". On the other hand, if the user model doesn't fit the system image, this affects the experience negatively. One user commented: "I was frustrated because I kept on wanting to drag and it won't drag".

In addition, we noted that the amount of physical activities that users engage with in addition to the timing in which they occurred during the course of the flow of interaction affected the experience. For example, during the course of the study one of the participants complained about the necessity of frequent zooming, and when asked to elaborate on whether or not this was distracting he said, "It depended on what I was trying to do, if it was a natural break in between things...it wasn't [distracting] but if I was zoomed in say [author_1] and I want to go and take a look at [author_2] I got to zoom all the way out, then zoom back in, so yeah that was a little distracting." Here the user is indicating that if the zooming action occurs while he was in the middle of a thinking trail which is associated with accomplishing a specific task, then the physical activity of zooming affected his experience negatively by distracting him from accomplishing that task. Hence, this tends to indicate that for a positive experience to arise, the design of the InfoVis tool must allow for an uninterrupted flow of interaction. Ensuring the usability of the tool is essential; however, there is more to the experience than mere usability.

\subsubsection{Insight}

Insight is gained from the data, which is associated with the visual representation. Users must be able to understand the meaning of these representations, in addition to being able to identify patterns. For example, when one of the participants was asked to compare his experiences of using the visualization with his experience using other tools that he would normally employ to accomplish similar tasks such as, Google Scholar, ACM, or CiteSeer, he said that using the visualization, was "...way way easier to get an overview of things really quickly". In our case, the visual representations were mostly clear and users didn't have problems understanding the visual cues.
In addition to the visual representation and the insight that participants gained, we noted that the completeness of the dataset was also crucial, and might have a negative effect on the experience. This was something that we had not anticipated prior to this study. In our case, the users were interacting with a prototype that visualizes a limited dataset. This dataset was incomplete in certain areas. For example, the citation trails of some seminal papers would stop abruptly, or in certain cases there were missing abstracts. This affected the feelings that the users had of the system and consequently their overall experiences. As in the case of usability, the inability of the tool to portray insight in certain areas affects the experience, particularly while the users are engaged in a sensemaking process. This, we believe is due to the fact that the flow of interaction is broken. Hence, we believe that it is crucial for the datasets used in the evaluation processes to be as realistic as possible. However, this is not always possible as it is very difficult to find clean complete datasets.

Usability and insight are things that cannot be pre-calculated. It is true that there are principles that when applied will deliver usable interfaces with well-understood visual cues, yet this is not guaranteed. People are different, their backgrounds and past experiences affects their experiences. This was quite apparent from our analysis and hence we identified background as another crucial category that affects the experience.

\subsubsection{Background}

Users' backgrounds affect the InfoVis experience. People rely on models that they create of their past experiences interacting with interfaces and apply these when interacting with new interfaces as seen when we talked about usability. In addition, people's backgrounds and knowledge affect the way that they gain insight of the domain. During the course of the study one of the users said, "I wanted to scan more and I couldn't scan more text at once." This was because when he clicked on a research paper, the tool showed an overview of the paper which included information such as: title, authors, and references. If the user wanted to see more details then he needed to right-click on the paper and select the details option from the popup menu. He, however, relying on his past experiences wanted to be able to access the text directly with the least amount of effort. On the other hand, given the same functionality, another user commented: "I thought that it was really neat that you can just right click and get the abstract right away so you can go through someone [an author] and at a glance".

In addition to the effects that the background might have on usability and insight, the user's background might have a direct effect on user's satisfaction of the tool. During the course of the study, when a user was asked to comment on her experiences she said: "just because they are in little boxes doesn't necessarily mean it is doing anything different that you would do a list". Here it can be seen that the user could not see the benefits that the tool had to offer and hence didn't really engage with the tool.

\subsubsection{User satisfaction}

Finally, we identified the category of user satisfaction. We believe that this is the goal of the user's InfoVis experience. Users reached the satisfaction stage if they were able to sense the benefits that the tool offered them, which in most cases was related to the speed with which they were able to gain insight. In other words, it was related to the ability of the tool to assist users in engaging with the internalization process. This finding was expected, however there were a few very interesting findings that 
were not apparent prior to this study which are related to increasing users' satisfaction with the tool. These are related to the concreteness of the results and the personalization of the experience. Both of these were in some way affected by the "marking tool" explained earlier. Although this tool is very simple, it gained astonishing popularity among users and was directly relevant to user satisfaction.

\section{Concreteness of the results}

The "marking tool" allowed users to see the results of their sensemaking efforts. Some users referred to this as a kind of a "mind map" which is interesting, since mind maps are a way of externalizing internalized understanding. This indicates that people were looking for ways visualize their internalized understanding of the externalized information.

In addition it was very common that participants asked whether or not they were able to store the results of their domain related sensemaking efforts. One user commented, "What I would've really liked to have had is to have a running list of what a marked list was ... but as well as being able to see them visually ...it would have felt satisfying that that was my thing that I was going to walk away with, that that was my package kind of thing". Users wanted to have a tangible sense of the efforts that they have accomplished. We think that this relates back to the importance of the completeness of the sense making activity, in that users need to store the results of their actions as they do when interacting with literature in the real world. This indicates that the task of the visualization need not stop at mere externalization but need to take the user from the beginning to the end of the experience.

\section{Personalization}

The marking tool is very simple. Interestingly, although a simple functionality, it was used differently by different users in ways that we did not anticipate. Users adapted it to their own needs. Some used it to further filter the data: "Being able to go through them and put my own, so its like a two step filter so the system filters and then I filter [using the marking tool] that was really useful". Others used it for navigating through the domain as landmarks: "I won't feel lost I can go back".

This is in keeping with the notion of design for appropriation as explained by Dix [7], where design features should be flexible and not rigid, in a way that allows users to employ them for various unanticipated purposes. We believe that applying this design principle when it comes to designing the functionality of the InfoVis tools is crucial, since it will allow for greater personalization, which as we felt from the study is greatly needed. For example, one of the users when asked about their use of the author citation map said, "I wasn't able to delete items from the map it became very complicated very quickly ... I think if it had been possible to delete items like a mind map then I think that I probably would have continued and made it more expansive I even would have wanted to walk away with a printout that sort of thing". This comment, like many others during the course of the study, strongly indicated a need for greater personalization. From our analysis, it appears that people want to be able to see their internalization of the data.

\section{Discussion}

Usability is not enough when it comes to evaluating InfoVis tools. Usability is designed for evaluating the interface, and hence is not solely suitable for evaluating InfoVis tools. InfoVis is not just an interface; it is an experience that needs to be fully understood in order to be effectively evaluated. Qualitative methods, as described, are appropriate for understanding such experiences. As a result, we applied these methods, specifically GT, to understand the InfoVis experience. From this study we identified categories that could not have otherwise been identified. These categories were explained at a high-level, as more data is currently being analyzed since the study is still on going.

The InfoVis user experience can be seen as a personal journey that takes the user from the externalization to internalization, from syntax to semantics. From our study we have learned that any of the components that make up the visualization experience can have either a positive or a negative effect on the users' overall satisfaction with the tool. This is not only related to the visual cues, but also related to the design rationale which should fit with the characteristics of the domain, in addition to the input devices and its associated physical activities. The aim is to reduce such activities as much as possible so as not to interfere with users' InfoVis sensemaking activity.

Users are not equal; they have past experiences and knowledge that distinguishes them as individuals. These differences have great influence on the InfoVis experience. If users are not able to see the benefits of the tool as soon as they interact with it then this affects their feelings towards the tool. Hence, we strongly believe that it is crucial for the evaluation process to be as realistic as possible. This ranges from the design of the high-level evaluation task to the completeness of the dataset used and its close correspondence to similar real world situations. For example, literature in real situations is reachable online with a simple click of a button. Users interacting with our tool were not able to access the actual papers; the inability of our tool to provide a complete literature experience affected the overall satisfaction. Hence, our goal is to integrate this tool with other literature tools to provide a more complete literature experience.

In addition to the realism of the experience, we identified that InfoVis experiences needs greater personalization. Personalization does not merely reflect the ability of the users to change the colors and shapes of the visual entities to fit their aesthetic pleasures. Personalization, on the other hand, suggests designing the InfoVis tools in a way that allows users to be able to overlay their own views over the data's externalization. Since users are different, it is difficult to add personalization features that will fit all users' needs. As a result, we call for the adoption of the principle of designing for appropriation, such that features are designed to be flexible enough for users to be able to employ them as they see fit, as the "marking tool" that our visualization provides.

Analyzing the interviews of the first 6 participants assisted us in better understanding the InfoVis experience, and allowed us to identify additional features and improvements that need to be added to our tools' design. We are in the process of analyzing the remainder of the interviews in addition to the screen recordings. We strongly believe that by generating theory that we, as a community, will be able to build and feed into each others work and hopefully be able to generate a theory of InfoVis interaction that will influence the design and evaluation of better InfoVis tools.

\section{Conclusion}

InfoVis tool are not just tools they are experiences. Before we can design or evaluate these experiences they need to be well understood. Qualitative methods have the power in unraveling these experiences and theorizing them. We have demonstrated the 
benefits that such methods have in identifying key components that were not previously discussed in the InfoVis domain. InfoVis is not only dependent on the visual representations but also reliant on the users, the domain, the interface and the physical activities. We also pointed to the importance of designing for greater personalization and touched on the means with which this can be tackled. We strongly believe that, in order for InfoVis tools to be able to reach the users, users' needs should no longer be neglected. Users have the information we need in order for us to evolve. We just need to start listening.

\section{REFERENCES}

[1] Baskerville, R.L., 1999. Investigating Information Systems with Action Research. Communications of the Association for Information Systems. 2(3es), p.4.

[2] Beyer, H. \& Holtzblatt, K., 1997. Contextual Design: Defining Customer-Centered Systems, Morgan Kaufmann.

[3] Card, S.K., Mackinlay, J.D. \& Shneiderman, B., 1999. Readings in Information Visualization: Using Vision to Think, Morgan Kaufmann Publishers Inc. San Francisco, CA, USA.

[4] Chin, J.P., Diehl, V.A. \& Norman, K.L., 1988. Development of an Instrument Measuring User Satisfaction of the HumanComputer Interface. In Proceedings of SIGCHI conference on Human Factors. Washington, D.C., United States: ACM, p. 213-218.

[5] Craft, B. \& Cairns, P., 2006. Using Sketching to Aid the Collaborative Design of Information Visualisation SoftwareA Case Study, In Human Work Interaction Design: Designing for Human Work, Springer Boston, Volume (221), p. 103-122,.

[6] Creswell, J.W., 1998. Qualitative Inquiry and Research Design: Choosing Among Five Traditions, Sage Publications.

[7] Dix, A., Designing for Appropriation. In Volume 2 Proceedings of the 21st BCS HCI Group Conference. Lancaster, UK, p. 28-30.

[8] Faisal, S., Cairns, P. \& Blandford, A., 2006. Developing User Requirements for Visualizations of Literature Knowledge Domains. In Proceeding of the $10^{\text {th }}$ International Conference on Information Visualisation (IV'06). London, UK: IEEE, p. 264-269.

[9] Faisal, S., Cairns, P. \& Blandford, A., 2006. Subjective Information Visualizations. In Proceedings of the V\&I Workshop on Combining Visualization and Interaction to Facilitate Scientific Exploration and Discovery, BSC HCI Group Conference. London, UK.

[10] Faisal, S., Cairns, P. \& Blandford, A., 2007. Building for Users not for Experts: Designing a Visualization of the
Literature Domain. In Proceedings of the $11^{\text {th }}$ International Conference on Information Visualsation (IV'07). Zurich, Switzerland: IEEE, p. 707-712.

[11] Ke, W., Borner, K. \& Viswanath, L., InfoVis04 Contest MSAccess Database, Indiana University, School of Library and Information Science and School of Informatics. Available at: http://ella.slis.indiana.edu/ lviswana/iv04-contest.mdb.

[12] Kuhn, T.S., 1970. The Structure of Scientific Revolutions, University of Chicago Press.

[13] MacEachren, A.M., 1995. How Maps Work: Representation, Visualization, and Design, York: Guilford Press.

[14] Mazza, R. \& Berrè, A., Focus Group Methodology for Evaluating Information Visualization Techniques and Tools. In Proceedings of the $11^{\text {th }}$ International Conference Information Visualisation (IV'07). IEEE, p. 74-80.

[15] Morse, E., Lewis, M. \& Olsen, K., 2000. Evaluating Visualizations: Using a Taxonomic Guide. International Journal of Human-Computers Studies, 53(5), p.637-662.

[16] Neilsen, J., 1995. Advances in HCI Vol 5, NJ: Albex, Norwood.

[17] Norman, D.A., 1988. The Psychology of Everyday Things, Basic Books New York.

[18] Saraiya, P., North, C. \& Duca, K., 2005. An Insight-Based Methodology for Evaluating Bioinformatics Visualizations. IEEE Transactions on Visualization and Computer Graphics, 11(4), p.443-456.

[19] Seo, J., \& Shneiderman, B., 2006. Knowledge Discovery in High-Dimensional Data: Case Studies and a User Survey for the Rank-by-Feature Framework. Visualization and Computer Graphics, IEEE Transactions on, 12(3), p.311322.

[20] Shneiderman, B. \& Plaisant, C., 2006. Strategies for Evaluating Information Visualization Tools: MultiDimensional In-Depth Long-Term Case Studies. In $A V I$ Workshop on Beyond Time and Errors: Novel Evaluation Methods for Information Visualization (BELIV'06). Venice, Italy: ACM, p. 1-7.

[21] Spence, R., Information Visualization: Design for Interaction Second Edition, Edinburgh Gate, Harlow, Essex, England. ACM Press Books.

[22] Strauss, A.L. \& Corbin, J.M., 1998. Basics of Qualitative Research: Techniques and Procedures for Developing Grounded Theory, Sage Publications.

[23] Ware, C., 2004. Information Visualization: Perception for Design, Morgan Kaufmann. 